\title{
Front Matter: Volume 9452
}

, "Front Matter: Volume 9452," Proc. SPIE 9452, Infrared Imaging Systems: Design, Analysis, Modeling, and Testing XXVI, 945201 (29 May 2015); doi: 10.1117/12.2184311

SPIE. Event: SPIE Defense + Security, 2015, Baltimore, MD, United States 


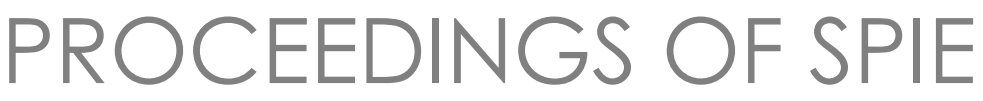

\section{Infrared Imaging Systems: Design, Analysis, Modeling, and Testing XXVI}

Gerald C. Holst

Keith A. Krapels

Editors

21-23 April 2015

Baltimore, Maryland, United States

Sponsored and Published by

SPIE 
The papers included in this volume were part of the technical conference cited on the cover and title page. Papers were selected and subject to review by the editors and conference program committee. Some conference presentations may not be available for publication. The papers published in these proceedings reflect the work and thoughts of the authors and are published herein as submitted. The publisher is not responsible for the validity of the information or for any outcomes resulting from reliance thereon.

Please use the following format to cite material from this book:

Author(s), "Title of Paper," in Infrared Imaging Systems: Design, Analysis, Modeling, and Testing XXVI, edited by Gerald C. Holst, Keith A. Krapels, Proceedings of SPIE Vol. 9452 (SPIE, Bellingham, WA, 2015) Article CID Number.

ISSN: 0277-786X

ISBN: 9781628415681

\section{Published by}

\section{SPIE}

P.O. Box 10, Bellingham, Washington 98227-0010 USA

Telephone +1 3606763290 (Pacific Time) · Fax +1 3606471445

SPIE.org

Copyright @ 2015, Society of Photo-Optical Instrumentation Engineers.

Copying of material in this book for internal or personal use, or for the internal or personal use of specific clients, beyond the fair use provisions granted by the U.S. Copyright Law is authorized by SPIE subject to payment of copying fees. The Transactional Reporting Service base fee for this volume is $\$ 18.00$ per article (or portion thereof), which should be paid directly to the Copyright Clearance Center (CCC), 222 Rosewood Drive, Danvers, MA 01923. Payment may also be made electronically through CCC Online at copyright.com. Other copying for republication, resale, advertising or promotion, or any form of systematic or multiple reproduction of any material in this book is prohibited except with permission in writing from the publisher. The CCC fee code is 0277-786X/15/\$18.00.

Printed in the United States of America.

Publication of record for individual papers is online in the SPIE Digital Library.

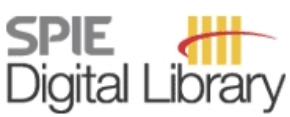

SPIEDigitalLibrary.org

Paper Numbering: Proceedings of SPIE follow an e-First publication model, with papers published first online and then in print. Papers are published as they are submitted and meet publication criteria. A unique citation identifier (CID) number is assigned to each article at the time of the first publication. Utilization of CIDs allows articles to be fully citable as soon as they are published online, and connects the same identifier to all online, print, and electronic versions of the publication. SPIE uses a six-digit CID article numbering system in which:

- The first four digits correspond to the SPIE volume number.

- The last two digits indicate publication order within the volume using a Base 36 numbering

system employing both numerals and letters. These two-number sets start with 00, 01, 02, 03, 04, $05,06,07,08,09,0 A, 0 B \ldots$.. 0Z, followed by 10-1Z, 20-2Z, etc.

The CID Number appears on each page of the manuscript. The complete citation is used on the first page, and an abbreviated version on subsequent pages. 


\title{
Contents
}

\author{
vii Authors \\ ix Conference Committee
}

\section{SESSION 1 TESTING AND SYSTEMS}

945202 Blackbox imager characterization, from measurements to modeling range performance (Invited Paper) [9452-1]

945203 Characterizing a high resolution color display performance using a Prichard photometer [9452-2]

945204 Validating machine vision MRT performance against trained observer performance for linear shift invariant sensors [9452-3]

945205 Advanced E-O test capability for Army Next-Generation Automated Test System (NGATS) [9452-4]

945206 Signal intensity transfer function determination on thermal systems with stray light or scattering present [9452-5]

945207 Measuring noise equivalent irradiance of a digital short-wave infrared imaging system using a broadband source to simulate the night spectrum [9452-6]

945208 Statistical analysis of target acquisition sensor modeling experiments [9452-7]

945209 Modeling analysis metrics for panoramic sensors [9452-8]

SESSION 2 TARGETS, BACKGROUNDS AND ATMOSPHERICS

$94520 \mathrm{~A}$ Simulation of a polarized laser beam reflected at the sea surface: modeling and validation [9452-9]

9452 OB Detector integration time dependent atmospheric turbulence imaging simulation [9452-10]

9452 OC Automatic parameter estimation for atmospheric turbulence mitigation techniques [9452-12]

9452 OD Atmospheric turbulence and sensor system effects on biometric algorithm performance [9452-13]

9452 OE An adaptive tracker for ShipIR/NTCS [9452-14] 
9452 OF Simulation-based sensor modeling and at-range target detection characterization with MUSES (Invited Paper) [9452-15]

9452 OG Probability of detection using ShipIR/NV-IPM [9452-16]

$9452 \mathrm{OH} \quad$ A TRM4 component for the Night Vision Integrated Performance Model (NV-IPM) [9452-17]

9452 Ol An independent survey of monochrome and color low light level TV cameras [9452-18]

9452 0J Depth of field in modern thermal imaging [9452-19]

\section{SESSION 4 MODELING II}

9452 OK Optimum viewing distance for target acquisition (Invited Paper) [9452-20]

$9452 \mathrm{OL} \quad$ A noise model for the design of a compressive sensing imaging system [9452-21]

9452 OM Block randomization versus complete randomization of human perception stimuli: is there a difference? [9452-22]

945200 Comparative performance of large-format MWIR and LWIR systems in NV-IPM [9452-43]

SESSION $5 \quad$ MODELING III

9452 OP Legacy modeling and range prediction comparison: NV-IPM versus SSCamIP and NVTherm [9452-24]

$9452 \mathrm{OQ} \quad$ Custom component generation in the night vision integrated performance model [9452-25]

9452 OR Investigating the validity of the networked imaging sensor model [9452-26]

9452 OS Model of Large-format EO-IR sensor for calculating the probability of true and false detection and tracking for moving and fixed objects [9452-27]

9452 OT Face acquisition camera design using the NV-IPM image generation tool [9452-28]

SESSION 6 HWIL

9452 OW Development of an ultra-high temperature infrared scene projector at Santa Barbara Infrared Inc. [9452-31]

9452 OX Test pixels for high-temperature infrared scene projection [9452-32]

9452 oY Thermal resolution specification in infrared scene projectors [9452-33]

$94520 Z$ Spectral homogenization techniques for the hyperspectral image projector [9452-34] 
945210 Advances in iterative non-uniformity correction techniques for infrared scene projection [9452-35]

945211 Computing the total atmospheric refraction for real-time optical imaging sensor simulation [9452-36]

945212 Memory efficient atmospheric effects modeling for infrared scene generators [9452-37]

\section{POSTER SESSION}

$945214 \quad$ New night vision goggle gain definition [9452-38]

945215 Application and validation of a S/W developed for IR signal simulation by using the measured data from a moving test ship [9452-39]

945216 Contrast performance modeling of broadband reflective imaging systems with hypothetical tunable filter fore-optics [9452-40] 
Proc. of SPIE Vol. $9452945201-6$

Downloaded From: https://www.spiedigitallibrary.org/conference-proceedings-of-spie on 26 Apr 2023 Terms of Use: https://www.spiedigitallibrary.org/terms-of-use 


\section{Authors}

Numbers in the index correspond to the last two digits of the six-digit citation identifier (CID) article numbering system used in Proceedings of SPIE. The first four digits reflect the volume number. Base 36 numbering is employed for the last two digits and indicates the order of articles within the volume. Numbers start with 00, 01, 02, 03, 04, 05, 06, 07, 08, 09, OA, OB...0Z, followed by 10-1Z, 20-2Z, etc.

Burdette, Edward M., 00

Burks, Stephen D., 02, 04, 06, 0Q

Burks, Thomas J., 06

Byrd, Kenneth A., OD

Calhoun, Seth, $\mathrm{OX}$

Choi, Hee-Sue, OT

Choi, Jun-Hyuk, 15

Coffey, Aubrey, OX

Coffey, Kevin R., OX

Curran, Allen R., OF

Danielson, Tom, OW, OX, OY, 10

Deaver, Dawne M., 08

Dein, Edward, OX

Dobbins, Christopher L., 00

Doe, Joshua M., 04

Driggers, Ronald G., 09

Du Bosq, Todd W., OB, OH, OL

Eppeldaver, George P., 14

Errea, S., 05

Espinola, Richard L., OD

Flug, Eric A., 03

Franks, Gregory, OW, OX, OY, 10

Franks, John, 0J

Fredricksen, Christopher J., OX

Friedman, Melvin H., OR

Furxhi, Orges, 09

Goodwin, Scott H., OW, OX

Green, John R., 07

Grigor, J., 05

Grossman, Stanley I., OS

Haefner, David P., 02, 06, 0Q

Han, Kuk-II, 15

Hillberry, Logan E., $\mathrm{OZ}$

Hixson, Jonathan G., 03, OP

Hodgkin, Van A., 16

Holst, Gerald C., OG, OK

Howell, Christopher L, OT

Kaur, Balvinder, 03

Kavak, Çağlar, 12

Kelmelis, Eric, OC

Kelly, Kevin F., OL

Kim, Dong-Geon, 15

Kim, Tae-Kuk, 15

King, D. F., 05

Korb, Andrew R., OS

Kozacik, Stephen, OC

Krapels, Keith, 09

Lannon, John M., OW, OX

Larason, Thomas C., 14
LaVeigne, Joseph D., OW, OX, OY, 10

Leonard, Kevin R., OD

Matis, G., 05

McHugh, Steve, 05, OW

McKechnie, J., 05

Moyer, Steve, 08, 0M

Namazi, Nader, OL

Nehmetallah, Georges, OL

Nehring, Brian, 05, 10

Olson, Jeff T., 03

Olson, Richard F., 11

Özsaraç, Seçkin, 12

Packard, Corey D., OF

Paolini, Aaron, OC

Peale, Robert E., OX

Podobedov, Vyacheslav B., 14

Potvin, Guy, OD

Preece, Bradley L., 09, OI, OL

Prewarski, Marcus, 10

Ramaswamy, Srinivasan, OE, OG

Repasi, Endre, $\mathrm{OB}, \mathrm{OH}$

Reynolds, Joseph P., OH, OI, OT

Rice, Joseph P., $0 Z$

Richardson, Philip I., 03

Robinson, Timothy, 07

Rynes, Peter L., OF

Saur, Nicholas E., OF

Schuster, Norbert, OJ

Schwenger, Frédéric, $O A$

Teague, James R., 00

Teaney, Brian P., 02, 04, OH, OP, OQ

Tomkinson, David M., OI, OP

Trewick, Stephen, OX

Uhl, Elizabeth R., OM

Vaitekunas, David A., OE, OG

Wood, Samuel B., 00 
Proc. of SPIE Vol. $9452945201-8$

Downloaded From: https://www.spiedigitallibrary.org/conference-proceedings-of-spie on 26 Apr 2023 Terms of Use: https://www.spiedigitallibrary.org/terms-of-use 


\title{
Conference Committee
}

\author{
Symposium Chair
}

Nils R. Sandell Jr., Strategic Technology Office, DARPA

(United States)

Symposium Co-chair

David A. Logan, BAE Systems (United States)

Conference Chairs

Gerald C. Holst, JCD Publishing (United States)

Keith A. Krapels, U.S. Army Night Vision \& Electronic Sensors Directorate (United States)

Conference Program Committee

Gary H. Ballard, U.S. Army Research, Development and Engineering Command (United States)

Gisele Bennett, Georgia Institute of Technology (United States)

Piet Bijl, TNO Defence, Security and Safety (Netherlands)

James A. Buford Jr., U.S. Army Research, Development and Engineering Command (United States)

James A. Dawson, Dynetics, Inc. (United States)

Ronald G. Driggers, St. Johns Optical Systems (United States)

Richard L. Espinola, U.S. Army Night Vision \& Electronic Sensors

Directorate (United States)

David P. Forrai, L-3 Communications Cincinnati Electronics

(United States)

Jonathan G. Hixson, U.S. Army Night Vision \& Electronic Sensors

Directorate (United States)

Alan Irwin, Santa Barbara Infrared, Inc. (United States)

Eddie L. Jacobs, University of Memphis (United States)

Terrence S. Lomheim, The Aerospace Corporation (United States)

R. Lee Murrer Jr., Millennium Engineering and Integration Company (United States)

Teresa L. Pace, L-3 Communications (United States)

Hector M. Reyes, Raytheon Company (United States)

Endre Repasi, Fraunhofer-Institut für Optronik, Systemtechnik und Bildauswertung (Germany)

Joseph P. Reynolds, U.S. Army Night Vision \& Electronic Sensors

Directorate (United States)

Bernard M. Rosier, ONERA (France)

Michael A. Soel, FLIR Systems, Inc. (United States)

Andrew W. Sparks, L-3 Sonoma EO (United States) 
Curtis M. Webb, Northrop Grumman Electronic Systems

(United States)

\section{Session Chairs}

1 Testing and Systems

Alan Irwin, Santa Barbara Infrared, Inc. (United States)

Curtis M. Webb, Northrop Grumman Electronic Systems (United States)

2 Targets, Backgrounds and Atmospherics

Richard L. Espinola, U.S. Army Night Vision \& Electronic Sensors Directorate (United States)

Endre Repasi, Fraunhofer-Institut für Optronik, Systemtechnik und Bildauswertung (Germany)

Bernard M. Rosier, ONERA (France)

3 Modeling I

Keith A. Krapels, U.S. Army Night Vision \& Electronic Sensors

Directorate (United States)

Gisele Bennett, Georgia Institute of Technology (United States)

Piet Bijl, TNO Defence, Security and Safety (Netherlands)

James A. Dawson, Dynetics, Inc. (United States)

Jonathan G. Hixson, U.S. Army Night Vision \& Electronic Sensors Directorate (United States)

Andrew W. Sparks, L-3 Sonoma EO (United States)

4 Modeling II

Ronald G. Driggers, St. Johns Optical Systems (United States)

David P. Forrai, L-3 Communications Cincinnati Electronics (United States)

Eddie L. Jacobs, The University of Memphis (United States)

Terrence S. Lomheim, The Aerospace Corporation (United States)

Andrew W. Sparks, L-3 Sonoma EO (United States)

5 Modeling III

Teresa L. Pace, L-3 Communications (United States)

Hector M. Reyes, Raytheon Company (United States)

Joseph P. Reynolds, U.S. Army Night Vision \& Electronic Sensors

Directorate (United States)

Michael A. Soel, FLIR Systems, Inc. (United States)

Andrew W. Sparks, L-3 Sonoma EO (United States)

6 HWIL

James A. Buford Jr., U.S. Army Research, Development and

Engineering Command (United States)

R. Lee Murrer Jr., Millennium Engineering and Integration Company (United States)

Gary H. Ballard, U.S. Army Research, Development and Engineering Command (United States) 